\title{
Chemically Consistent Evolutionary Synthesis Modelling of Galaxies
}

\author{
Uta Fritze $^{1} \&$ Thorsten Tepper García ${ }^{2}$ \\ ${ }^{1}$ University of Hertfordshire, UK, e-mail: ufritze@star.herts.ac.uk \\ ${ }^{2}$ Universität Göttingen, Germany, e-mail: tepper@astro.physik.uni-goettingen.de
}

\begin{abstract}
We present our chemically consistent GALEV Evolutionary Synthesis models for galaxies and point out differences to previous generations of models and their effects on the interpretation of local and high-redshift galaxy data.
\end{abstract}

Keywords. galaxies: evolution, galaxies: high-redshift, galaxies: photometric redshifts

GALEV evolutionary synthesis models start from a gas cloud of primordial abundances and form stars according to a given Star Formation History (SFH) and a stellar Initial Mass Function (IMF). SFH and IMF are the only free parameters. Models use a data base of stellar input physics to calculate both the chemical evolution of the gas and the spectral evolution of the stellar component from the onset of SF until the present time. Coupled to a cosmological model, as specified by $\mathrm{H}_{0}, \Omega_{\mathrm{m}}, \Omega_{\lambda}$, GALEV models also describe the redshift evolution of chemical abundances and spectral properties. The SFHs are the basic parameter, our default IMF is Salpeter, other IMFs are investigated for comparison. Simple Stellar Populations (SSPs) like star clusters are formed without chemical enrichment in a single short burst $\left(\sim 10^{5} \mathrm{yr}\right)$, all of their stars have the same metallicity and age. Galaxies are composite stellar populations with SFHs extended in time. For undisturbed galaxy types, simplified Star Formation Rates (SFRs) are used for the respective spectral types: $\operatorname{SFR}(\mathrm{t}) \sim \mathrm{e}^{-\mathrm{t} / \tau_{*}}$ with $\tau_{*}=1$ Gyr for classical Es, and SFRs coupled to the evolving gas content $\mathrm{G}, \mathrm{SFR}(\mathrm{t}) \sim \mathrm{G}(\mathrm{t})$, with characteristic timescales increasing from S0s to Sc's and const. SFRs for Sd's, similar to the Bruzual \& Charlot, Pegase, and Starburst99 models. These SFHs in principle go back to Sandage (86) and are constrained in detail be agreement between models and local galaxy samples/templates. Interacting galaxies and hierarchical assembly are described via the starbursts they trigger in our simplified 1-zone models without dynamics.

Input physics for the stars and the gas includes stellar evolutionary tracks/isochrones (Padova vs. Geneva), stellar model atmosphere spectra and Lick absorption features, gaseous emission in terms of lines and continuum, and stellar yields (PNe, SNII, SNIa) for individual elements ( $\mathrm{He}, \mathrm{C}, \mathrm{N}, \mathrm{O}, \ldots, \mathrm{Fe})$. Complete sets of input physics are available for 5 different metallicities $[\mathrm{Fe} / \mathrm{H}]=-1.7 \ldots+0.4$ for solar scaled abundances. GALEV model output is the time evolution of Color - Magnitude - Diagrams (CMDs) and the time and redshift evolution of integrated quantities: spectra $(90 \AA \ldots 160 \mu \mathrm{m})$, emission line strengths, luminosities and colors in various filter systems (Johnson, HST, Washington, Strömgren, . . ) ) Lick absorption features $\left(\mathrm{Mg}_{2}, \mathrm{Mgb}, \mathrm{Fe} 5270, \mathrm{Fe} 5335\right.$, TiO1, $\mathrm{TiO} 2, \ldots$ ), galaxy (or star cluster) masses: gas and stars, M/L, ISM abundances for the above elements calculated from a modified version of Tinsley's equations including SNIa contribution à la Matteucci (carbon deflagration white dwarf binaries). All the pieces of input physics depend significantly on metallicity and so does, of course, the output. This is best seen on SSPs for different metallicities: low metallicity stellar populations are hotter, brighter, bluer, have stronger UV and ionising fluxes, hence stronger 
emission lines, than high metallicity populations (Schulz et al. 02). Emission line ratios of heavy element lines behave in a complex way as a function of metallicity, therefore we use empirical line ratios (cf. Anders \& Fritze 03). Together with stellar lifetimes, stellar yields for individual elements also show a complex metallicity dependence with stellar yield ratios significantly deviating from solar ratios at low metallicities, e.g. $[\mathrm{Mg} / \mathrm{Fe}]$ increases with decreasing metallicity. Different SFHs - short and burst-like vs. mild and constant - lead to different abundance ratios in the gas between elements with different nucleosynthetic origin, as e.g. $[\mathrm{C} / \mathrm{O}],[\mathrm{N} / \mathrm{O}]$, or $[\alpha / \mathrm{Fe}]$. $\mathrm{C}$ and $\mathrm{N}$ originate in intermediate mass stars, Fe has important SNIa contributions, both leading to a delayed production with respect to the SNII products $\mathrm{O}, \mathrm{Mg}$, etc. A threshold in metallicity, below which SNIa may be inhibited (Kobayashi et al. 98), would further enhance this effect. Via the SFH, galaxy evolution and stellar evolution become intimately coupled. In principle, stellar evolutionary tracks, yields and model atmospheres are required not only for various metallicities (and He contents), but also for various abundance ratios. However, no complete grids of stellar input physics are available yet for varying abundance ratios $[[\mathrm{C} / \mathrm{O}],[\mathrm{N} / \mathrm{O}],[\alpha / \mathrm{Fe}], \ldots]$. Our chemically consistent (= cc) GALEV models follow the evolution of ISM abundances together with the spectrophotometric properties of galaxies and account for the increasing initial metallicity of successive generations of stars by using for each stellar generation the set of input physics appropriate for its initial metallicity. Models thus appropriately account for the observed broad stellar metallicity distributions (typically $\leqslant 2 \mathrm{dex}$ ) in galaxies (e.g. Rocha-Pinto \& Maciel 98, Sarajedini \& Jablonka 05, Harris \& Harris 00) as well as for the increasing importance of low metallicity (sub-)populations in local late-type and dwarf galaxies (Kobulnicky \& Zaritsky 99) and in galaxies at higher redshift, in particular the intrinsically fainter ones less accessible to spectroscopy but detected in huge numbers in deep multi-band imaging surveys (Mehlert et al. 02, Pettini et al. 02, Tremonti et al. 04).

Models clearly show that light contributions from various age and metallicity subpopulations are very different at different wavelengths, whence the enormous analytical power of multi-band deep photometry over a long wavelength basis and whence significantly different metallicities are expected to be seen at different wavelengths. In spirals the youngest stars with the highest metallicities clearly dominate at the shortest wavelength with the result that the stellar metallicity seen in $\mathrm{U}$ is higher than in $\mathrm{V}$ and much higher than in $\mathrm{K}$ where older and less metal rich stars dominate the light. In ellipticals, in turn, the metallicity of stars dominating in $\mathrm{K}$ is about 1.8 and 2 times higher than that of stars dominating the light in V and U (cf. Möller et al. 97, Bicker et al. 04). This fact has bearing on any attempts to calibrate new stellar metallicity indicators, e.g. in the NIR. They will inevitably show different stellar metallicities than their optical counterparts because of the composite nature of stellar populations in galaxies and the fact that different stellar age and metallicity subpopulations dominate the light at different wavelengths. Models also show that even classical initial collapse elliptical galaxies feature broad stellar metallicity distributions, in good agreement with observations of the stellar metallicity distributions in the halo of NGC 5128 (Harris \& Harris 00). Therefore, elliptical galaxies cannot be adequately described/analysed by simple (=single metallicity, single age) stellar population models. Models also show how the stellar metallicity distributions and the light contributions of stellar subpopulations of various metallicities have evolved with time. E.g., at a redshift $\mathrm{z} \sim 1$, when galaxies have about half their present age, no solar metallicity stars are yet formed in average luminosity Sb-type galaxies, the bulk of their stars have $1 / 4$ to $1 / 5$ of solar metallicity (see Bicker et al. 04 for details). Were these galaxies at $z \geqslant 1$ analysed with solar metallicity models, their SFRs, metallicities, 
photometric masses etc. would be seriously in error, as are already quantities derived for local late-type and dwarf galaxies unless their subsolar metallicities are appropriately taken into account. In Bicker \& Fritze (05), we showed that SFRs derived from $\mathrm{H}_{\alpha}$ and [OII] for nearby low metallicity galaxies like IZw18 or SBS 0335 are overestimated by factors 2 and 3, respectively, when using the standard calibrations from Kennicutt (98) or Gallagher et al. (89) that are valid for solar or near-solar metallicities. Accurate SFRs can only be derived together with metallicities from either spectroscopy or multi-band imaging.

To obtain from the time evolution of gas abundances their redshift evolution only requires a transformation between age and redshift, directly given by the cosmological parameters and an assumed redshift at which SF started. Our comparison of the chemical evolution of the ISM in spiral galaxies from cc GALEV models with observed element abundances from Keck HIRES spectroscopy in the neutral gas of Damped Ly $\alpha$ Absorbers (DLAs) in Lindner et al. (99) has shown a) that DLAs observed in ample numbers to redshifts $\mathrm{z}=4$ and higher can well be the progenitors of local spiral galaxy types Sa ... Sd with model abundances naturally connecting the low DLA abundances (typically $1 / 100$ to few tenths of solar at redshifts $\mathrm{z}=2-4$ ) to HII region abundances in local spirals, b) that the DLA phase is a normal transition phase in the life of a spiral in the sense that early-type spirals reaching high metallicities at the same time have consumed their gas to an extent where they drop out of the high column density DLA samples, c) that protospirals at $\mathrm{z}=2-4$ already must have had $50-100 \%$ of their present-day total mass, albeit largely in the form of gas, in agreement with dynamical DLA mass estimates from rotation velocities by Prochaska \& Wolfe (97), Wolfe et al. (05), and d) that DLA galaxies have faint luminosities and low SFRs in agreement with recent VLT (non-)detections. The most important conclusion from this comparison between DLA abundances and our spiral models is that it confirms the average SFHs of our spiral models over a lookback time to $\mathrm{z}>4$ of more than $90 \%$ of the Hubble time.

To transform the time evolution of spectral quantities into their respective redshift evolution requires an evolutionary correction (accounting for the fact that distant galaxies are seen in younger evolutionary stages), a cosmological correction (accounting for redshifting and dimming of the galaxy light on its way through the expanding universe) and including the effect of attenuation of the galaxy light at wavelengths below Ly $\alpha$ due to the cumulative effect of intergalactic neutral hydrogen HI (cf. Madau 95, Bershady et al. 99, Tepper García 06). With all these effects properly taken into account, our cc GALEV models give a fair description of the redshift evolution of galaxies in the Hubble Deep Field North with photometric redshifts from Sawicky \& Yee (97). They thus allow to identify progenitors at high redshift of the different local galaxy types and to study their respective mass assembly and chemical enrichment histories. The agreement between spectrophotometric observations and models out to redshifts $\mathrm{z} \sim 4$ also confirms our model SFHs (Bicker et al. 04). A number of galaxies at $\mathrm{z} \geqslant 1$ bluer than our bluest Sd model and some galaxies at $0.5 \leqslant z \leqslant 2.5$ redder than our reddest undisturbed $\mathrm{E}$ model are readily explained in terms of starburst and post-starburst models, respectively, as shown in Fritze \& Bicker (06a). We found starbursts to be frequent at $\mathrm{z}>1$ and very strong at high redshifts, increasing the stellar mass by $\geqslant 30 \%$.

In Bicker \& Fritze (in prep.) we calculated a large grid of cc GALEV models for various galaxy types $(\mathrm{E}, \ldots, \mathrm{Sd}$, starbursts, and post-starbursts) and all redshifts and developed an analysis tool that, on the basis of a $\chi^{2}$ method, compares the observed Spectral Energy Distribution (SED) of a galaxy to the model grid to find not only the best but all acceptable fits, and determine galaxy type and photometric redshift, SFR and age, gaseous and stellar masses and metallicities, all including their respective 
1- $\sigma$ uncertainties. Comparison with the subsample of HDF-N galaxies with spectroscopic redshifts shows that our photometric redshifts agree with the spectroscopic ones to $\leqslant 5 \%$ (Fritze \& Bicker 06b). Doing this kind of analysis on the basis of cc GALEV model spectra as opposed to using a locally observed set of galaxy spectra has the advantage to consistently account for evolutionary and cosmological effects including attenuation and for the increasing importance of subsolar metallicity stars in distant younger galaxies. Comparison with the same analysis done on a grid of GALEV models using solar metallicity input physics only shows that in this case acceptable solutions are also found, but often with wrong (type, redshift) combinations and that ages are underestimated by typically a factor of 2 , photometric masses tend to be overestimated by factors up to 5 and more, and SFRs overestimated by factors 2 and higher (Tepper García \& Fritze b in prep.).

So far, the chemical and spectral aspects of galaxy evolution have been coupled consistently in our GALEV models, next steps will be the consistent inclusion of dust with the dust content coupled to the evolving gas content and metallicity and accounting for the relative distributions of dust and stars in different galaxy types (see Möller et al. 01a, b, c for an encouraging first attempt) and the coupling of cc GALEV models with dynamical galaxy models including gas, stars, SF and feedback on the relevant (pc to $\mathrm{kpc}$ ) scales. The coupling of GALEV models into a cosmological structure formation simulation was first attempted by Contardo et al. (98), albeit by that time with still insufficient resolution, in particular for SF and feedback.

\section{References}

Anders, P., \& Fritze-v.A., U. 2003, A\&A 401, 1063.

Bershady, M.A., Charlton, J.C., \& Geoffroy, J.M. 1999, ApJ 518, 103.

Bicker, J., \& Fritze-v.A., U. 2005, A\&A 443, L19.

Bicker, J., Fritze-v.A., U., Möller, C.S., \& Fricke, K.J. 2004, A\&A 413, 37.

Contardo, G., Steinmetz, M., \& Fritze-v.A., U. 1998, ApJ 507, 497.

Fritze-v.A., U., Bicker, J. 2006, A\&A 454, 67.

Fritze-v.A., U., Bicker, J., \& Cunow, B. 2006, IAU Symp. 232, 508.

Gallagher, J.S., Hunter, D.A., \& Bushouse, H. 1989, AJ 97, 700.

Harris, G.L.H., \& Harris, W.E. 2000, AJ 120, 2423.

Kennicutt, R.W. 1998, ARAA 36, 189.

Kobayashi, C., Tsujimoto, T., Nomoto, K., et al. 1998, ApJ 503, L155.

Kobulnicky, H.A., \& Zaritsky, D. 1999, ApJ 511, 118.

Lindner, U., Fritze-v.A., U., \& Fricke, K.J. 1999, A\&A 341, 709.

Madau, P. 1995, ApJ 441, 18.

Mehlert, D., Noll, S., Appenzeller, I., et al. 2002, A\&A 393, 809.

Möller, C.S., Fritze-v.A., U., \& Fricke, K.J. 1997, A\&A 317, 676.

Möller, C.S., Fritze-v.A., U., \& Calzetti, D. 2001a, Ap\&SS 277, 601.

Möller, C.S., Fritze-v.A., U., Calzetti, D., \& Fricke, K.J. 2001b, IAU Symp. 204, 413.

Möller, C.S., Fritze-v.A., U., Fricke, K.J., \& Calzetti, D. 2001c, Ap\&SS 276, 799.

Pettini, M., Ellison, S.L., Bergeron, J., \& Petitjean, P. 2002, A\&A 391, 21.

Prochaska, J.X., \& Wolfe, A.M. 1997, ApJ 487, 73.

Rocha-Pinto, H.J., \& Maciel, W.J. 1998, A\&A 339, 791.

Sandage, A. 1986, A\&A 161, 89.

Sarajedini, A., \& Jablonka, P. 2005, AJ 130, 1627.

Sawicky, M., \& Yee, H.K.C. 1997, AJ 113, 1.

Schulz, J. Fritze-v.A., U., Möller, C.S., \& Fricke, K.J. 2002, A\&A 392, 1.

Tepper García, T. 2006, MNRAS, 369, 2025.

Tremonti. C.A., Heckman, T.M., Kauffmann, G., et al. 2004, ApJ 613, 898.

Wolfe, A.M., Gawiser, E., \& Prochaska, J.X. 2005, ARAA 43, 861. 


\section{Discussion}

Robert Rubin: Can your GALEV model predict Ne/S ratio as a function of metallicity?

Thorsten Tepper-Garcia: No, I think not. 